\title{
A Survey about Intelligent Solutions for Autonomous Vehicles based on FPGA
}

\author{
$1^{\text {st }}$ Ashraf Kasem \\ Institute of Automation and Infocommunication \\ University of Miskolc \\ Miskolc, Hungary \\ kasem.ashraf.sabet@student.uni-miskolc.hu \\ $3^{\text {rd }}$ József Vásárhelyi \\ Institute of Automation and Infocommunication \\ University of Miskolc \\ Miskolc, Hungary \\ vajo@mazsola.iit.uni-miskolc.hu
}

\author{
$2^{\text {nd }}$ Ahmad Reda \\ Institute of Automation and Infocommunication \\ University of Miskolc \\ Miskolc, Hungary \\ autareda@uni-miskolc.hu \\ $4^{\text {th }}$ Ahmed Bouzid \\ Institute of Automation and Infocommunication \\ University of Miskolc \\ Miskolc, Hungary \\ qgebouzid@uni-miskolc.hu
}

\begin{abstract}
Safe driving and reducing the number of accidents victims have been the main motivations for researchers and automotive companies for decades. Today, humanity is very close to make the old dream of fully autonomous vehicles a reality, thanks to the rapid spread of AI (artificial intelligence) and the evolution of semiconductor technologies. But the real problem here is the increasing demand for computational power and that of course will increase power requirements, hence it will not be suitable for autonomous driving applications. GPU is not suitable for solving this problem due to its power consumption as well as heat generation. On the other hand, CPU also does not satisfy the performance requirements. For the above condition, FPGA (Field Programmable Gate Array) has drawn attention as a hardware accelerator since it features high performance with low power consumption. This paper reviews the common solutions involving artificial intelligence implemented on FPGA for autonomous vehicle applications. Research, development, and current trends related to the topic are emphasized.
\end{abstract}

Keywords- FPGA, AI, Autonomous Vehicles, HW Acceleration, Coprocessing

\section{INTRODUCTION}

FPGA (Field-Programmable Gate Array) is an integrated circuit designed to be configured by the designer after its manufacture, as it contains a large array of programmable blocks and reconfigurable interconnects which give it high flexibility in many applications. The history of FPGA goes back to 1985 when Ross Freeman and Bernard Vonderschmitt invented the viable field-programmable gate array. Because of its high performance and low power consumption, many companies have started to use FPGA to accelerate the performance of their systems. One of the most interesting fields that FPGA is starting to take part in is the industry of autonomous vehicles. Table I shows that the automotive industry is the third most important sector in the market. Autonomous vehicles that drive us instead of us driving them will be a reality soon and that will increase road safety since accidents related to impaired driving could be reduced as cars cannot get drunk or be distracted by a text message or by another passenger. Thus, if we remove humans from the equation, autonomous vehicles will make the roads safer.

Autonomous vehicles are equipped with multiple sensors such as cameras, radars, and LiDARs, to perceive the surrounding environment.

These sensors generate huge data, hence real-time processing this data requires high performance computing systems. Instead of using CPUs and GPUs for implementing AI (Artificial Intelligence), FPGAs are adopted since they feature high performance with low power consumption. This article presents the latest solutions for autonomous vehicles involving AI implemented on FPGAs.

TABLE I. GLOBAL FPGA MARKET SHARE ESTIMATION IN 2019 [1]

\begin{tabular}{cccccccc}
\hline Field & Telecom. & Ind. & Auto. & Consum. & Milit. & DP & Others \\
\hline $\begin{array}{c}\text { Market } \\
\text { Share }\end{array}$ & 32 & 18 & $\mathbf{1 6}$ & 14 & 9 & 7 & 4 \\
\hline \multicolumn{8}{c}{$\begin{array}{l}\text { Telecom. (Telecommunication), Ind. (Industrial), Auto. (Automotive), } \\
\text { Consum. (Consumer Electronics), Milit. (Military \& Aerospace), DP (Data Processing) }\end{array}$}
\end{tabular}




\section{IMPLEMENTATION METHODS}

Few years ago, FPGA designs were quasi-exclusively performed by electrical engineers since the implementation tools were low level of abstraction. It was hard to design complex solutions using HDL (Hardware Description Language), thus the increase of the design productivity gap. The considerable progress of the implementation tools including reusable IPs (Intellectual Properties) increases the popularity of FPGAs allowing a large variety of engineers (from hardware to software engineers) to develop FPGAbased systems.

There are many ways to deploy algorithms on FPGAs:

HDL: Using VHDL (Very High-Speed Integrated Circuit Hardware Description Language), an implementation of a vehicle collision avoidance solution has been proposed considering a PID (Proportional-Integral-Derivative) controller based on fuzzy logic [2]. A HW/SW (Hardware/Software) codesign to implement an ADAS (Advanced Driver-Assistance Systems) was presented in [3]. The hardware accelerator, performing an ANFIS (Adaptive Neuro-Fuzzy Inference System) clustering in the PL (Programmable Logic) side of the Zynq SoC (System on Chip), was implemented in VHDL.

HLS (C/C++): Vivado HLS (High Level Synthesis) has been used in [4] to deploy $\mathrm{C} / \mathrm{C}++$ code into a Xilinx FPGA to implement a white line detector where the single line search method gives better results than Hough transform in terms of processing time.

Python: One of the latest trends on FPGA development is the use of Python. The authors in [5] presented an implementation on FPGA/Python of lane line detection system. The results show that the execution time of FPGA/Python is 6.34 times faster than OpenCV considering the implementation of median blur, adaptive threshold, and accumulation of Hough transform.

MicroBlaze: The classification of traffic signs is an important aspect in autonomous driving. In [6] is presented an implementation method for traffic sign detection using HW/SW codesign. The classification is processed on a MicroBlaze soft core and the preprocessing is performed on hardware.

PS+PL vs. MB+IP: Xilinx SoCs are made of two major parts, PS (Processing System) and PL, which can be exploited to have HW/SW codesign based systems. The authors in [7] show that implementing a TSR (Traffic Sign Recognition) system on a Xilinx SoC gives better results than its implementation in an MicroBlaze + IP based system

XSG (Xilinx System Generator): is a powerful development tool to implement FPGA designs as it is suitable for DSP (Digital Signal Processing) applications. The authors in [8] used XSG to model, simulate and implement a traffic signs detection system which presents promising results.

HDL coder: is a tool provided by MATLAB that helps to deploy MATLAB/SIMULINK models into FPGAs after automatic generation of HDL code. An MPC (Model Predictive Control) controller has been implemented on SoC using HDL Coder for automated steering of vehicles [9].

\section{BACKGROUND}

An autonomous driving system is implemented on hybrid computational technologies GPU-FPGA, where the GPU's primary job is self-driving and the FPGA's job is to perform some subtasks such as pedestrian and traffic lights detection [10]. An end-to-end solution for the design of self-driving cars based on Xilinx PYNQ-Z2 board is proposed in [11]. The most important characteristic of this design is the presence of the DPU (Data Process Unit) that accelerates the deep learning process. FPGA is faster than the CPU and consumes less power than the GPU when it comes to accelerate the CNN (Convolutional Neural Networks) process [12]. The proposed architecture permits to reduce the execution time and energy consumption comparing to the CPU.

The accuracy and delay of autonomous driving systems have a major impact on how the vehicle handles the surrounding environment. To make the delay, resulting from data inputs, deterministic a solution is proposed in [13] considering bypassing the CPU from the input data path as shown in Fig. 1.

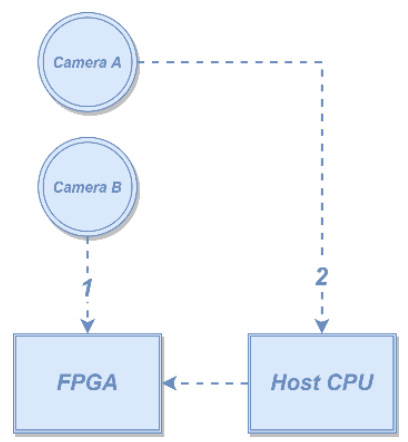

Fig. 1. Line 1 has more deterministic delay from line 2 [13]

The biggest challenge when implementing AI algorithms in FPGAs is the difficulty of the hardware design. H. Bingo [14] proposes a solution to this problem applying PYNQ board which allows the use of Python for its wide range libraries, especially in AI and Image processing. O. Changsong and Y. Jong-min [15] discuss the most popular technologies to accelerate deep learning processes for autonomous cars industry. Some steps are introduced to avoid collisions in the air for UAV (Unmanned Aerial Vehicle) using four cameras based on FPGA SoC (System on Chip) [16]. The collision avoidance algorithm has already been implemented in GPU-based system, but the solution is not practical as it requires high energy. Road segmentation is a very important process in self-driving cars which identifies and describes the drivable parts of the roads. The problem with this process is the need of high computational resources. A model based on FPGA is suggested in [17] to perform a realtime and low power road segmentation. The authors suggest the use of LiDAR (Light Detection and Ranging) than the use of traditional cameras since they suffer from image unclarity in poor lighting conditions. FPGA is used for scene perception based on CNN. It took about $16.9 \mathrm{~ms}$ to accomplish a CNN operation using Xilinx UltraScale XCKU115 FPGA.

In self-driving cars environment, if there is more than one car starting from the same place and going to the same destination, the decision-making system will choose the same 
optimal path for all cars. There will be one busy road/track and other uncrowded as shown in Fig. 2. To solve this issue, paper [18] proposes a solution implemented on FPGA applying game theory. This solution relies on direct communication between vehicles to apply game theory instead of using the cloud. The author of [19] relies on the use of two FPGA board. PYNQ-Z1 to deal with detecting obstacles and pedestrians by implementing light weight BNN (Binarized Neural Networks) and Zynq- Xc7Z010 to recognize traffic lights and detect road lines. The two FPGA boards were connected to each other using Ethernet to be able to communicate. Execution time is reduced and accuracy in identifying obstacles is increased by this architecture.

The authors of the article [20] suggest a hybrid method in which the computing problem of machine learning is solved. The machine learning process should contain two phases: Training phase and inference phase. A machine learning platform was built so that the training phase takes place in the GPU and inference phase in FPGA as shown in Fig. 3. This platform is user friendly and has greatly improved performance. In [21] a tool is provided to accelerate and improve the performance of applying Deep Neural Network (DNN) on FPGA. The proposed design used two different methods to manage the on-chip FPGA memory.

The first one is to store the complete feature maps on the chip in order reduce the complexity of the data movements. The second technique is to automatically generate buffers to overcome the data shortage when the processes are fetching data from the external memory. The proposed design provides real-time performance for processing HD videos and deliver a higher efficiency (up to 4.35x) than the GPU-based solutions. FpgaConvNet is a framework and tool to automatically implement CNN on FPGA (presented in [22]). The provided tool applies analytical synchronous data flow and the parallelism processing across the $\mathrm{CNN}$ layers without constraints on the model size. FpgaConvNet provides hardware optimization by considering the latency as the core

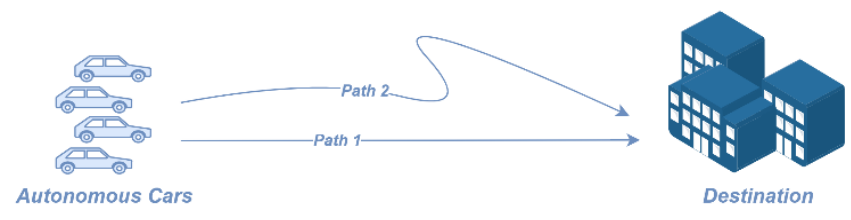

Fig. 2. All autonomous cars will choose path 1 since it is the optimal path and that will make it crowded [18]

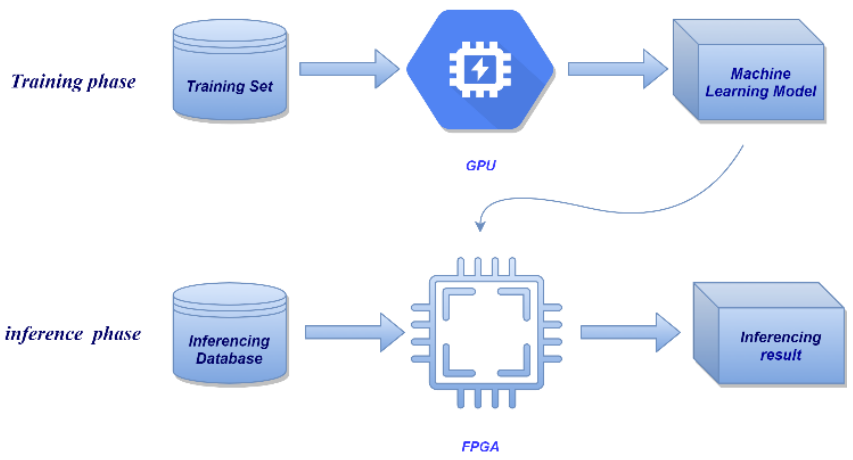

Fig. 3. The architecture of the hybrid machine learning platform [20] of optimization and achieves a performance up to $6.65 \mathrm{x}$ comparing with the optimized GPUs. The paper also suggests a method to be used by the CascadeCNN which has two stages as shown in Fig. 4. In the first one, a quick classification is performed based on very low precision processor. In the second phase, the estimation of the prediction confidence is performed. The samples which are wrongly classified are reprocessed using a high precision processor to reach the required accuracy level and that improves the performance up to $48 \%$.

The article [23] presented multi-core processing units capable of increasing the level of automation in Urban Air Mobility (UAM) aircraft by using a parallel sensor fusion structure to increase accuracy and safety during landing. These processing units are based on FLS (Fuzzy Logic System), Which interacts with several sensors that have different in frequency ranges and spatially separated. The proposed method was designed and tested using MATLAB and VHDL on Intel Altera OpenVINO FPGA board. The proposed FLS processing units are capable of achieving a computational speed of 25.3 GOPS (Giga Operations per Seconds).

The architecture offers five Hybrid Obstacle Avoidance (HOA) units to control height as shown in Fig. 5. Landing decisions will be based on the fused information coming from these units. Each node contains two different types of sensors each one works on a different frequency spectrum that will increase immunity to noise.

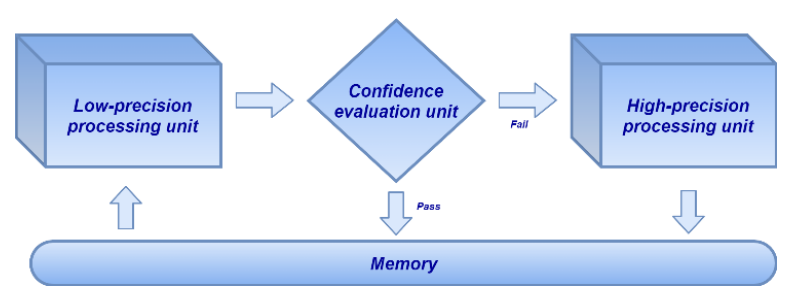

Fig. 4. CascadeCNN's high-level architecture [22]

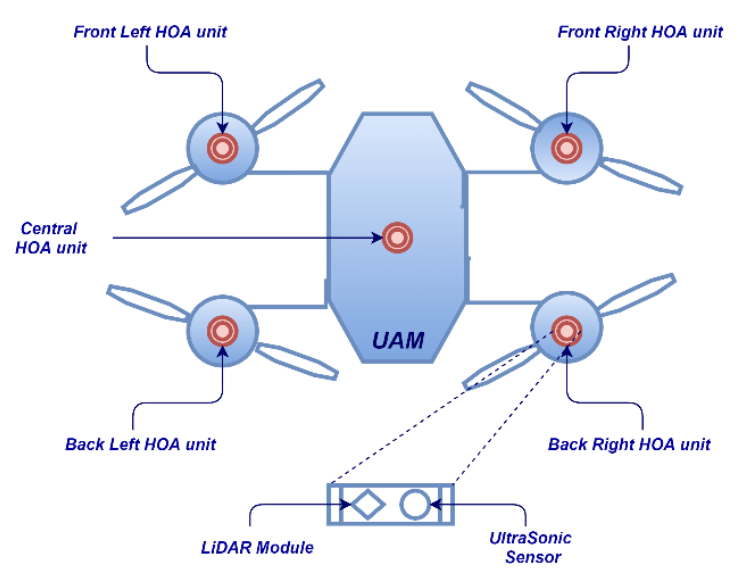

Fig. 5. HOA units constellation on the bottom Side of an UAM [23] 
One of the advantages of this design is that the FLS core does not depend on any external memory, thus increasing the security against cyberattacks, in addition to the low power consumption reaching less than $20 \mathrm{~mW}$ when working at the maximum frequency of $283 \mathrm{MHz}$. The need to recognize and process sequential data such as video streaming has made LSTM (long short-term memory) networks an important requirement for AI models. However, this LSTM needs high memory requirements, and this will cause many challenges in time sensitive applications.

Paper [24] presents an FPGA-based method for optimizing LSTM functionality in a critical-time environment. The proposed architecture gives a similar output in quality compared to a traditional LSTM, but in terms of speed it is $415 \mathrm{x}$ faster. This method proposes an approximate computing scheme that enables the calibration of the QoR (Quality of Result) in exchange for an increase in performance. This model approximates the weight arrays in a neural network using low-rank SVD (Singular-Value Decomposition) in addition to the distribution of weights arrays based on the importance of their components. These techniques allow to restructure the calculations for LSTM and design computing system that carries out the most important operations first, to get peak QoR within time constraints. Table II resumes recent implementation results of different algorithms that can be useful for autonomous vehicles solutions. The implementations are performed in FPGA (or SoC), and some are performed in GPU for comparison. It is clearly noticeable that the power performances provided by the FPGA are significantly better than in GPU. However, in terms of time performance, both hardware targets present close results that depend on the size of the processed images and the implemented algorithm. CPU based implementations have not been considered in the Table II since they show low performances in all cases.

\section{CONCLUSION}

In the present paper, we introduced the latest proposed FPGA-based solutions to accelerate AI processes dedicated to autonomous vehicles. It is doubtless that reaching fully autonomous and safe cars requires overcoming many challenges, for instance real-time processing of the huge data coming from multiple sensors, considering the energy consumption in addition to the safety issues. Exploiting reconfigurable computational technologies significantly enhance the performance of the solutions that especially involve artificial intelligence and autonomous vehicles since they require parallel computing. Depending on the solutions discussed in the paper, we are about to witness fully autonomous and safe vehicles in the next few years considering embeddability requirements.

\section{ACKNOWLEDGMENT}

This research was supported by the European Union and the Hungarian State, co-financed by the European Regional Development Fund in the framework of the GINOP-2.3.4-152016-00004 project, aimed to promote the cooperation between the higher education and the industry.

TABLE II. COMPARISON OF DIFFERENT IMPLEMENTATIONS FOR IMAGE PROCESSING ALGORITHMS THAT ARE USEFUL FOR AUTONOMOUS VEHICLES

\begin{tabular}{|c|c|c|c|c|c|c|c|c|}
\hline Source & \multicolumn{2}{|l|}{ [25] } & \multicolumn{2}{|l|}{$[26]$} & \multicolumn{2}{|l|}{ [27] } & {$[28]$} & [29] \\
\hline Purpose & \multicolumn{2}{|c|}{ Canny Edge Detector } & \multicolumn{2}{|c|}{ STOP Sign Detection } & \multicolumn{2}{|c|}{$\begin{array}{l}\text { Speed-Limit Sign } \\
\text { Recognition }\end{array}$} & $\begin{array}{l}\text { Roberts Edge } \\
\text { Detector }\end{array}$ & Lane Detection \\
\hline Platform & $\begin{array}{l}\text { FPGA } \\
\text { (Intel Arria V } \\
\text { 5AGXFB3) }\end{array}$ & $\begin{array}{l}\text { GPU } \\
\text { (GeForce } \\
\text { GTX 580) }\end{array}$ & $\begin{array}{l}\text { SoC } \\
\text { (Xilinx } \\
\text { ZC706) }\end{array}$ & $\begin{array}{l}\text { GPU } \\
\text { (GRID } \\
\text { K520) }\end{array}$ & $\begin{array}{l}\text { FPGA } \\
\text { (Intel Arria } \\
10 \mathrm{GX} \text { ) }\end{array}$ & $\begin{array}{l}\text { GPU } \\
\text { (NVIDIA } \\
\text { GTX1060) }\end{array}$ & $\begin{array}{l}\text { SoC } \\
\text { (Intel Cyclone V } \\
\text { 5CSEMA6F31A7) }\end{array}$ & $\begin{array}{l}\text { FPGA } \\
\text { (Intel Stratix IV } \\
\text { EP4SGX230KF40C2) }\end{array}$ \\
\hline Resolution & \multicolumn{2}{|c|}{$3936 \times 3936$} & \multicolumn{2}{|c|}{$1920 \times 180$} & \multicolumn{2}{|c|}{$640 \times 240$} & $\begin{array}{l}481 \times 321 \\
\text { (grayscale) }\end{array}$ & $1024 \times 768$ \\
\hline $\begin{array}{l}\text { Processing Time } \\
\text { (ms/frame) }\end{array}$ & 64.16 & 59.94 & 7.9 & 24.3 & 33.3 & 30.3 & 0.85 & 5.4 \\
\hline Power (W) & 1.5 & 251 & 5.2 & 72 & 12.5 & 19 & 0.433 & - \\
\hline Frequency (MHz) & 242 & 772 & 100 & 745 & - & - & 100 & 200 \\
\hline Accuracy (\%) & - & - & 99.8 & 99.8 & 90 & 90 & 98.9 & - \\
\hline Frame rate (fps) & 15.58 & 16.68 & 126.58 & 41.17 & 30 & 33 & 1175 & 185.18 \\
\hline
\end{tabular}

\section{REFERENCES}

[1] "Field Programmable Gate Array Market Size, Share \& Trends Analysis Report by Technology (SRAM, Antifuse, Flash), by Application (Military \& Aerospace, Telecom), by Region, and Segment Forecasts, 2020 - 2027', Apr, 2020.

[2] I. Md Shabiul, et al. "Fuzzy based PID controller using VHDL for transportation application." International Journal of Mathematical Models and Methods in Applied Sciences 2.2 (2008): 143-147.

[3] M.C. Óscar, et al. "An FPGA-Based Neuro-Fuzzy Sensor for Personalized Driving Assistance." Sensors 19.18 (2019): 4011.

[4] W. Hiromichi, et al. "Development of a robot car by single line search method for white line detection with FPGA." 2018 International Conference on Field-Programmable Technology (FPT). IEEE, 2018.
[5] H. Koki, K. Wei, and H. Amano. "FPGA/Python co-design for lane line detection on a PYNQ-Z1 board." 2019 IEEE 13th International Symposium on Embedded Multicore/Many-core Systems-on-Chip (MCSoC). IEEE, 2019.

[6] S. Fynn, L. Gierke, and Michael Hübner. "FPGA based traffic sign detection for automotive camera systems." 2015 10th international symposium on reconfigurable communication-centric systems-on-chip (ReCoSoC). IEEE, 2015.

[7] H. Yan, et al. "Hardware/Software co-design of a traffic sign recognition system using zynq FPGAs." Electronics 4.4 (2015): 1062-1089.

[8] H. Rihab, A. Ben Abdelali, and Abdellatif Mtibaa. "Hardware implementation and validation of a traffic road sign detection and identification system." Journal of Real-Time Image Processing 15.1 (2018): 13-30. 
[9] A. Reda, A. Bouzid, and J. Vásárhelyi. "Model Predictive Control for Automated Vehicle Steering." Acta Polytechnica Hungarica 17.7 (2020).

[10] C. Hao et al. "A hybrid GPU+ FPGA system design for autonomous driving cars," IEEE International Workshop on Signal Processing Systems (SiPS), IEEE, 2019, pp. 121-126.

[11] T. Wu, W. Liu and Y. Jin, "An End-to-End solution to Autonomous Driving based on Xilinx FPGA," International Conference on FieldProgrammable Technology (ICFPT), IEEE, 2019, pp. 427-430.

[12] E. Adel, R. Magdy, S. Mohamed, M. Mamdouh, and H. Mostafa, “Accelerating Deep Neural Networks Using FPGA," IEEE International Conference on Microelectronics (ICM 2018), Sousse, Tunisia, pp. 176179, 2018.

[13] J. Ahmad and A. Warren, "FPGA based Deterministic Latency Image Acquisition and Processing System for Automated Driving Systems," IEEE International Symposium on Circuits and Systems (ISCAS), IEEE, 2018, pp. 1-5.

[14] H. Bingo, "Development of a control target recognition for autonomous vehicle using FPGA with Python," International Conference on FieldProgrammable Technology (FPT), IEEE, 2018, pp. 419-420.

[15] C.-s. Oh and J.-m. Yoon, "Hardware acceleration technology for deeplearning in autonomous vehicles," IEEE International Conference on Big Data and Smart Computing (Big-Comp), IEEE, 2019, pp. 1-3.

[16] F. Kóta, T. Zsedrovits and Z. Nagy, "Sense-and-avoid system development on an FPGA," International Conference on Unmanned Aircraft Systems (ICUAS), IEEE, 2019, pp. 575-579.

[17] Y. Lyu, L. Bai and X. Huang, "Real-time road segmentation using lidar data processing on an FPGA," IEEE International Symposium on Circuits and Systems (ISCAS), IEEE, 2018, pp. 1-5.

[18] S. Du, T. Huang, J. Hou, S. Song and Y. Song, "FPGA based acceleration of game theory algorithm in edge computing for autonomous driving," Journal of Systems Architecture 93, 2019, pp. 3339.

[19] W. Kaihie, K. Honda and H. Amano, "FPGA Design for Autonomous Vehicle Driving Using Binarized Neural Networks," International Conference on Field-Programmable Technology (FPT), IEEE, 2018, pp. 428-431.
[20] X. Liu, H. A. Ouni, A. Gherbia, Y. Lemieux and W. Li, "A Hybrid GPUFPGA-based Computing Platform for Machine Learning," the 9th International Conference on Emerging Ubiquitous Systems and Pervasive Networks, 2018, pp. 104-111.

[21] X. Zhang, J. Wang, C. Zhu, Y. Lin, J. Xiong, W. Hwu and D. Chen, "DNNBuilder: An Automated Tool for Building High Performance DNN Hardware Accelerators for FPGAs," IEEE International Conference on Computer-Aided Design, USA, 2018.

[22] S. Venieris, A. Kouris and C. Bouganis, "Deploying Deep Neural Networks in the Embedded Space," 2nd International Workshop on Embedded and Mobile Deep Learning, Germany, 2018.

[23] H. O. Ahmed, "25.3 GOPS Autonomous Landing Guidance Assistant System Using Systolic Fuzzy Logic System for Urban Air Mobility (UAM) Vehicles Using FPGA," Integrated Communications Navigation and Surveillance (ICNS) Conference, IEEE, USA, 2020

[24] A. Kouris, S. I. Venieris, M. Rizakis, and C.-S. Bouganis, "Approximate LSTMs for Time-Constrained Inference: Enabling Fast Reaction in Self- Driving Cars," IEEE Consumer Electronics Magazine, IEEE, 2020, pp. 11-26.

[25] P. P. Ricardo, et al. "A multi-resolution FPGA-based architecture for real-time edge and corner detection." IEEE Transactions on Computers 63.10 (2013): 2376-2388.

[26] X2 S. Weijing, et al. "An FPGA-based hardware accelerator for traffic sign detection." IEEE Transactions on Very Large Scale Integration (VLSI) Systems 25.4 (2016): 1362-1372.

[27] X3 Y. Matthew, et al. "Fpga versus gpu for speed-limit-sign recognition." 201821 st International Conference on Intelligent Transportation Systems (ITSC). IEEE, 2018.

[28] S. Douglas, et al. "An Analysis of the Implementation of Edge Detection Operators in FPGA." Anais Estendidos do X Simpósio Brasileiro de Engenharia de Sistemas Computacionais. SBC, 2020.

[29] G. Jungang, et al. "Real-time straight-line detection for XGA-size videos by Hough transform with parallelized voting procedures." Sensors 17.2 (2017): 270. 